\title{
Use of Layered Double Hydroxides to Remove Sunset Yellow FCF Dye from Aqueous Solution
}

\author{
ABD HAFIZ ABD MALEK ${ }^{1}$ and YAMIN YASIN ${ }^{* 2}$ \\ ${ }^{1}$ Department of Environmental Technology, Faculty of Applied Sciences, Universiti \\ Teknologi MARA 40450 Shah Alam, Selangor, Malaysia \\ ${ }^{2}$ International Education College, Universiti Teknologi MARA, 40450, Shah Alam, \\ Selangor, Malaysia \\ yamin961@salam.uitm.edu.my
}

Received 7 May 2012 / Accepted 19 May 2012

\begin{abstract}
The potential feasibility of layered double hydroxides for removal of sunset yellow dye FCF from aqueous solution was investigated. The effects of various experimental parameters such as contact time, solution $\mathrm{pH}$, dye concentrations and adsorbent dosage were investigated. The extent of sunset yellow FCF dye removal increased with the increased in contact time and amount of layered double hydroxides used however the percentage removal was decreased with the increased in $\mathrm{pH}$ and dye concentrations. Adsorption data was better fitted to the Langmuir isotherm. The results in this study indicated that layered double hydroxide was an attractive adsorbent for removing sunset yellow FCF dye from aqueous solution.
\end{abstract}

Keywords: Anionic dye, Adsorption, Clay, Layered double hydroxides

\section{Introduction}

Layered double hydroxide (LDH) is a class of anionic clays with high anion exchange capacities which can be used as an effective adsorbent for the removal of variety of anionic pollutant. Their empirical formula of $\mathrm{LDH}$ can be described by the formula $\left[\mathrm{M}^{2+} 1-\mathrm{x}\right.$ $\left.\mathrm{M}^{3+} \mathrm{x}(\mathrm{OH}) 2\right]^{\mathrm{x+}}\left(\mathrm{A}^{\mathrm{n}-)} \mathrm{x} / \mathrm{n} \cdot \mathrm{mH}_{2} \mathrm{O}\right.$ where $\mathrm{M}^{2+}$ and $\mathrm{M}^{3+}$ are metal cations, for example $\mathrm{Mg}^{2+}$ and $\mathrm{Al}^{3+}$ respectively, that occupy octahedral sites in the hydroxide layers, $A^{n-}$ is an exchangeable anion and $x$ is the ratio $\mathrm{M}^{3+} /\left(\mathrm{M}^{2+}+\mathrm{M}^{3+}\right)$. Carbonates are the interlayer anions in the naturally occurring mineral hydroxides, which is a member of this class of materials ${ }^{1,2}$. It is known that many substances in waste water, such as humic acid substances, dye in the effluents carry negative charges ${ }^{3}$. Species that carry negative charges account for a large part of water in the water systems ${ }^{4}$. The LDH's anion exchange ability, large surface area and regeneration ability ensures that this adsorbent can be effectively utilized in wastewater purification ${ }^{5}$.

Since inexpensive cost of synthesis, stability and variety of color, used of synthetic dyes increasingly in dyeing, paper and pulp, textile, rubber, plastics, cosmetics, pharmaceutical, food and other industrial applications ${ }^{6,7}$. This may lead to large volume of dye wastewaters ${ }^{8}$. Colour stuff discharged from these industries poses certain hazards and environmental problems. These coloured compounds are not only aesthetically displeasing but also inhibiting 
sunlight penetration into the stream and affecting aquatic ecosystem ${ }^{9}$. Dyes usually have complex aromatic molecular structures which make them more stable and difficult to biodegrade ${ }^{10}$. Furthermore, many dyes are toxic to some microorganisms and may cause direct destruction or inhibition of their catalytic capabilities ${ }^{11}$.

There are various conventional methods of removing dyes including coagulation and flocculation $^{5}$, oxidation or ozonation ${ }^{6}$ and membrane separation ${ }^{7}$. Nevertheless, due to their high cost and economic disadvantage, these methods are not widely used. Chemical and electrochemical oxidations, coagulation are generally not feasible on large scale industries. In distinction, an adsorption technique is by far the most versatile and widely used. The most common adsorbent materials are: alumina silica ${ }^{8}$, metal hydroxides ${ }^{9}$ and activated carbon $^{10}$. As proved by many researchers ${ }^{11,12}$, removal of dyes by layered double hydroxides is economically favourable and technically easier. The removal of organic species from discharge wastewater in order to reuse the water is clearly an environmental issue. Therefore, the purpose of this study is to evaluate the efficiency of removal sunset yellow FCF using anionic layered double hydroxides.

\section{Experimental}

Co-precipitation method was adapted to synthesis Mg-Al-NO 3 (LDH-MAN) in this work. In the preparation of LDH-MAN an aqueous solution of $\mathrm{Mg}\left(\mathrm{NO}_{3}\right)_{2} \cdot 6 \mathrm{H}_{2} \mathrm{O}(0.1 \mathrm{M})$ was added to $\mathrm{Al}$ $\left(\mathrm{NO}_{3}\right)_{3} .9 \mathrm{H}_{2} \mathrm{O}(0.025 \mathrm{M})$ to give $\mathrm{Mg}^{2+} / \mathrm{Al}^{3+}$ ratio, $\mathrm{R}=4$. Aqueous solution of $\mathrm{NaOH}$ and $\mathrm{Na}_{2} \mathrm{CO}_{3}$ $(2.0 \mathrm{M})$ were then added to the mixture dropwise, with vigorous stirring at room temperature and the $\mathrm{pH}$ was adjusted to $10.0 \pm 0.2$. The precipitate formed was aged at $70{ }^{\circ} \mathrm{C}$ in an oil bath shaker for 24 hours, cooled, centrifuged, washed till $\mathrm{pH} 7$ and dried in an oven for 24 hours. The resulting LDH-MAN was ground into powder and keep in sample bottles for further use and characterization.

X-ray diffraction (XRD) pattern of the sample powder was recorded on a PAN analytical X'Pert PRO Pan powder diffractometer using filtered $\mathrm{CuK} \alpha$ radiation at $60 \mathrm{Kv}$ and $60 \mathrm{~mA}$. The sample was mounted on PW18xx series holders and scans at 2-65 2/min at 0.003 steps. The basal spacing was determined via powder technique. Surface morphology and particle size distribution were studied using Carl Zeiss SMT Supra 40VP field emission scanning electron microscope at voltage of $5 \mathrm{kV}$.

\section{Preparation of dye solution}

Sunset yellow FCF dye purchased from Aldrich was used for the adsorption experiment. The dye stock solution was prepared by dissolving accurately weighed dye in distilled water to a concentration of $500 \mathrm{ppm}$. The experimental solution was obtained by diluting the dye stock solution in accurate proportions to different initial concentrations.<smiles>O=[N+]([O-])c1ccc(/N=N/c2c(O)ccc3cc(OS(=O)(=O)[O-])ccc23)cc1</smiles>

Figure 1. Structure of Sunset Yellow FCF dye

\section{Adsorption of sunset yellow FCF dye from aqueous solutions}

A sample of $0.25 \mathrm{~g}$ synthesized compounds was placed in a $100 \mathrm{~mL}$ Schott Duran bottles and $25 \mathrm{~mL}$ of $50-250 \mathrm{ppm}$ solution of dye was added to the bottles and agitated using water bath shaker operating at $150 \mathrm{rpm}$. The amount of dye adsorbed onto the compounds (\%) was calculated as: 


$$
\frac{C o-C t}{C o} \times 100 \%
$$

Where Co is the initial concentration (mM) of the samples (sunset yellow FCF) solution and $\mathrm{Ct}$ is the concentration at equilibrium at the time, $\mathrm{t}$. The solution is filter through $0.45 \mu \mathrm{m}$ Whatman syringe filter. The concentration of sunset yellow FCF dye was then measured by using Lambda 20 UV-Visible spectrophotometer. All the experiments were conducted in duplicate and controls were simultaneously carried out to ensure sorption was by adsorbents and not by the wall of the glassware. Adsorption isotherm was recorded over the concentration range 50-250 ppm of sunset yellow FCF dye in a series of $100 \mathrm{~mL}$ conical flask containing $50 \mathrm{~mL}$ solution of each concentration. A known amount of LDH-MAN was then added into the solution and conical flask was agitated.

\section{Results and Discussion}

\section{Characterization of layered double hydroxides}

The structure of layered double hydroxides (LDH-MAN) synthesized at different ratio and after adsorption of sunset yellow FCF were characterized and their XRD patterns was shown in Figure 2. As shown in the Figure, original layered double hydroxides indicate fairly good crystallinity. This proved by a d-spacing which recorded at $7.9 \AA$ that demonstrated general features of layered double hydroxides. The $d$-spacing shows the characteristic values for trigonal structures with a strong sharp and symmetrical peaks assigned to (003) and (006) planes, respectively. The interlayer spacing of the sample corresponding to the (006) plane was found to be $4.5 \AA$. The result is similar to the study reported previously ${ }^{5}$, in which the XRD pattern corresponds to that $\mathrm{LDH}$; the peaks are sharp signifying high crystallinity and increase as increase of LDH-MAN ratio. Layered double hydroxides (LDH-MAN) at ratio 4 was subsequently chosen as adsorbent for removal of sunset yellow FCF.

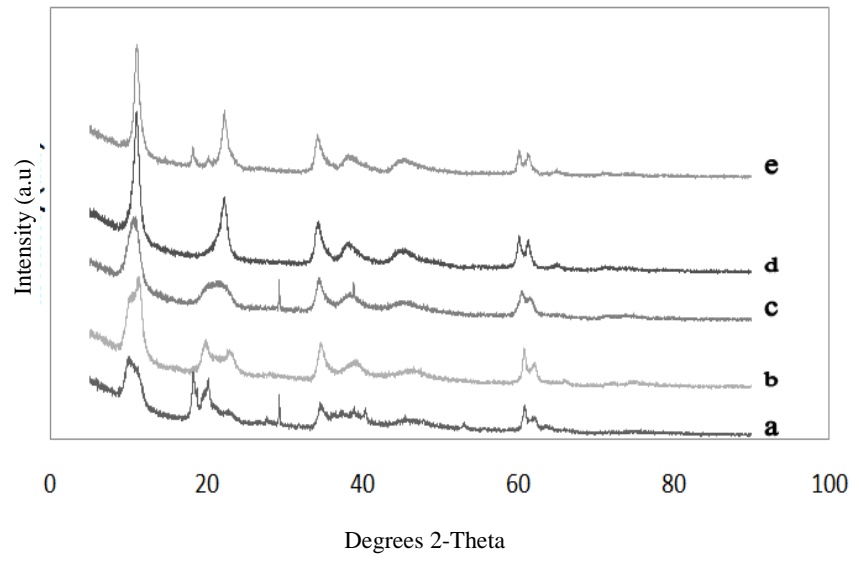

Figure 2. XRD of LDH-MAN at different Mg/Al ratio; (a) MAN 1 (b) MAN 2 (c) MAN 3 (d) MAN 4 and (e) MAN 4 after adsorption of sunset yellow FCF

The SEM image of LDH-MAN 4 in that shown in Figure 3 exhibits a regular layered structure with a very high aspect ratio. After adsorption of dye, the small flakes of sunset yellow FCF seem to be bound onto the LDH-MAN surface, as evidenced by small patches on the big LDH-MAN 4 sheets. This indicated of an ordered of sunset yellow FCF particles fill in the LDH-MAN's external and internal space and intercalated into the LDH-MAN surface. 

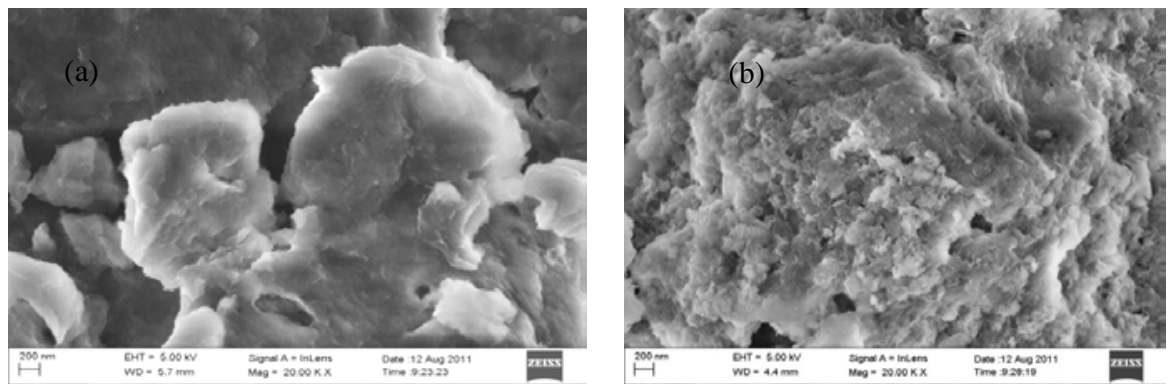

Figure 3. SEM image; (a) LDH-MAN 4 and (b) MAN 4 after adsorption of sunset yellow FCF

\section{Effect of contact time on adsorption}

The effect of contact time on removal of sunset yellow FCF was shown in Figure 4(a). The adsorption was carried out at times ranging from 30 minutes to 8 hours, under the same conditions of $298 \mathrm{~K}$ of temperature, $\mathrm{pH}$ of $6,0.5 \mathrm{~g}$ of LDH-MAN 4 and the concentration of sunset yellow FCF at $150 \mathrm{ppm}$ and $200 \mathrm{ppm}$, respectively. As shown in the Figure, the amounts of sunset yellow FCF dye removal increased rapidly within the initial 4 hours and remained almost unchanged after 5 hours indicating an equilibrium state. The removal of sunset yellow FCF dye exceeding 95\% was obtained but the percentage removal has not changed when incubation time was further increased. Among the factors that may have contributed to this are as follows; (i) an adsorption have achieved an equilibrium state (ii) as the adsorption progressed, sunset yellow FCF dye concentration decrease led to a fall in the degree of adsorption.

\section{Effect of $p H$ on adsorption}

The effect of $\mathrm{pH}$ study on the removal of dye was carried out within the range of $\mathrm{pH}$ from 2 to 12 . The adsorption of sunset yellow FCF was found to be a function of $\mathrm{pH}$ as shown in Figure 4(b).
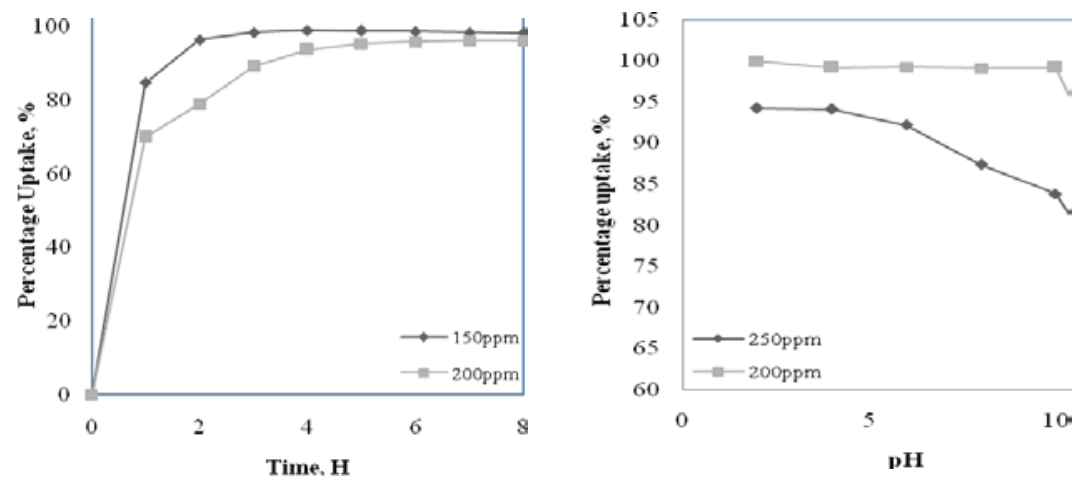

Figure 4. The effect of contact time (a) and $\mathrm{pH}$ (b) on the removal of sunset yellow FCF using LDH-MAN 4

The effect of $\mathrm{pH}$ on the removal of sunset yellow FCF was carried out at a fixed initial concentration of sunset yellow FCF (200 ppm and $250 \mathrm{ppm}$ ) and adsorbent dosage (LDHMAN 4) of $0.05 \mathrm{~g}$. As shown in the Figure, the percentage removal of sunset yellow FCF decreased with increasing $\mathrm{pH}$ and reached the maximum removal at $\mathrm{pH}$ of 4 . Further increased of $\mathrm{pH}$ will steadily decreased the percentage removal of sunset yellow FCF. Hence, the weak acid is the ideal condition for sunset yellow FCF dye removal using LDH-MAN 4. 


\section{Effect of adsorbent dosage on adsorption}

In order to investigate the effect of adsorbent mass on the removal of sunset yellow FCF dyes, a series of adsorption experiment was carried out with different adsorbent dosages from 0.01 to $0.05 \mathrm{~g}$ at initial dye concentrations of 50 to $250 \mathrm{ppm}$. Figure 5(a) shows the effect of adsorbent dosage on the removal of sunset yellow FCF by LDH-MAN 4. The results follow the expected pattern, in which the percentage removal increased with the increased of adsorbent dosage. As shown in the Figure, the percentage uptake of sunset yellow FCF increased rapidly and reached the maximum at $0.05 \mathrm{~g}$ dosages. These might be due to the increase of adsorbent sites available for dyes molecules and consequently better adsorption takes place. The result also shows that the percentage removal of sunset yellow FCF is higher at low concentration with the percentage removal exceeding 95\%. The percentage removal increased almost linearly with increased of dosage probably implying that the amount of LDH-MAN 4 sites also increased. This result was similar as reported by other researcher ${ }^{5,6}$ which consisted well in the paper.

\section{Effect of dye concentration on adsorption}

The effect of initial dye concentrations on the removal of sunset yellow FCF dye by LDHMAN 4 at different concentration was carried out at concentrations range from 50 to $250 \mathrm{ppm}$ with the used of adsorbent dosage range from 0.01 to $0.05 \mathrm{~g}$. The results as shown in Figure 5(b) pursue the expected pattern in which the removal of dye decreased with the increased in concentration of the dye solutions. This might be due to the total numbers of sorption sites available in LDH-Man 4 is constant.
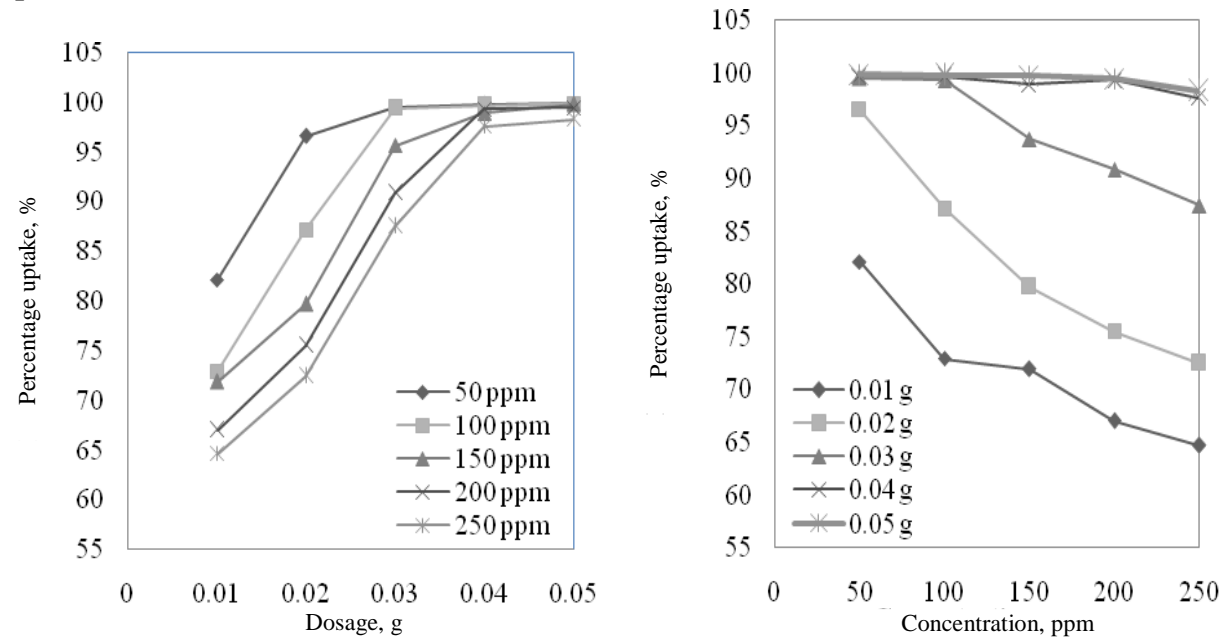

Figure 5. The effect of adsorbent dosage (a) and concentration of dye on the removal of sunset yellow FCF using LDH-MAN 4 (b)

\section{Adsorption isotherm}

In optimizing the use of adsorbents, Langmuir isotherm can be used as a model to describe the adsorption isotherm. The Langmuir equation is given as:

$$
\mathrm{C}_{\mathrm{e}} / \mathrm{q}_{\mathrm{e}}=1 / \mathrm{Q}_{\mathrm{o}} * \mathrm{~b}+\mathrm{C}_{\mathrm{e}} / \mathrm{Q}_{\mathrm{o}}
$$

Where Ce is the concentration of adsorbate solution at equilibrium ( $\mathrm{mgdm}^{-3}$ ), qe is the amounts of adsorbate adsorbed per mass of adsorbent ( $\mathrm{mg} / \mathrm{g}$ ), $\mathrm{b}$ is the equilibrium constant 
related to the sorption energy between the adsorbate and adsorbent $\left(\mathrm{dm}^{3} \mathrm{mg}^{-1}\right)$ and Qo is limiting amount of adsorbate that can be taken up per mass of adsorbent. Sorption equilibria provide fundamental physiochemical data evaluating the applicability of sorption process as a unit operation. Figure 6 shows the fitted equilibrium data for Langmuir isotherm expressions. It was shown that the equilibrium data fitted the Langmuir isotherm with high correlation coefficient of 0.999 was recorded. The higher correlation coefficients value for Langmuir isotherm indicated the monolayer adsorption of sunset yellow FCF on LDH-MAN 4. The calculated Langmuir isotherms constant for the maximum sorption capacity of sunset yellow, $\mathrm{Q}_{0}(\mathrm{mg} / \mathrm{g})$ was $142.86 \mathrm{mg} / \mathrm{g}$.

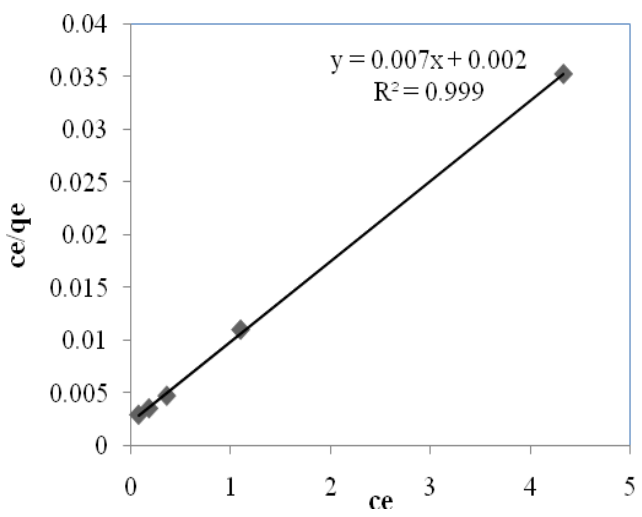

Figure 6. Langmuir isotherm plot for removal of sunset yellow FCF using LDH-MAN 4

\section{Conclusion}

From the present study, it can be concluded that layered double hydroxides can be used as potential adsorbent for the removal of sunset yellow FCF dye from aqueous solution.

In particular, it displayed the potential to effectively remove negatively charged species from aqueous solution. The adsorption of sunset yellow FCF dye increased with increased in contact time and exceeding 95\% removal after 5 hours. Moreover, it also shows the effective range of sunset yellow FCF removal was at $\mathrm{pH} 4$. Further decrease of $\mathrm{pH}$ could cause dissolution of LDH-MAN. Hence, the alkaline was a significant condition of dye adsorption as the surface became negatively charge. The percentage removal of sunset yellow FCF dye increased as increased in adsorbent dosage while decreased in removal as increased in concentration of the dye solution. The equilibrium data was well fitted to the Langmuir isotherm which confirming the monolayer coverage of sunset yellow FCF dye onto layered double hydroxides adsorbents.

\section{Acknowledgement}

The authors would like to thank the Ministry of Higher Education Malaysia for financial support for this work under Fundamental Research Grant Scheme.

\section{References}

1. Cavani F, Trifiro F and Vaccari A, Catal Today, 1991, 11, 173-301.

2. $\quad$ Arslanoglu F N, Kar F and Arslan N, J Food Eng., 2005, 71, 156-163.

3. Yasin Y, Abdul Malik A H, Sumari S M and Faujan B H A, R J Chem Environ., 2010, 14(1), 78-84. 
4. $\quad$ Yasin Y, Abdul Malik A H and Sumari S M, R J Chem Environ., 2011, 15(2), 876-882.

5. Yasin Y, Abd Malek A H and Sumari S M, Orient J Chem., 2010, 26(4), 1293-1298.

6. Yasin Y, Hussein M Z and Faujan B H A, Malaysian J Anal Sci., 2007, 11, 400 - 406.

7. Renmin G D, Yi L, Mei Y, Chao L and Yingzhi S, Dyes Pig., 2005, 64, 187-192.

8. Mao X Z, Li L, Hai H W and Zheng W, J Haz Mat., 2007, 149, 735-741.

9. Tsai W T, Chang CY, Lin M C, Chien S F, Sun H F and Hsieh M F, Chem., 2001, 45, 51-58.

10. Yasin Y, Abd Malek A H and Faujan B H A, J App Sci., 2010, 10(19), 2297-2303.

11. Santhy K and Selvapathy P, Bioresource Technol., 2006, 97, 1329-1336.

12. Órfão J J M, Silva A I M, Pereira J C V, Barata S A, Fonseca I M, Faria P C C and Pereira M F R, J Colloid Interface Sci., 2006, 296, 480-489. 